\title{
In silico mining identifies /GFBP3 as a novel target of methylation in
}

\section{prostate cancer}

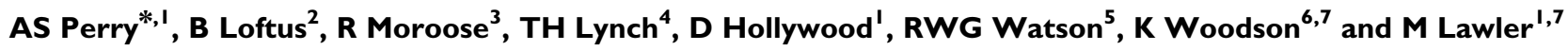 \\ 'Department of Haematology and Academic Unit of Clinical and Molecular Oncology, Institute of Molecular Medicine, St James's Hospital and Trinity \\ College Dublin, Ireland; ' ${ }^{2}$ Department of Histopathology, AMNCH and Trinity College Dublin, Ireland; ${ }^{3}$ Florida Hospital Cancer Center, Orlando, USA; \\ ${ }^{4}$ Department of Urology, St James's Hospital, Ireland; ${ }^{5}$ UCD School of Medicine and Medical Science, Conway Institute of Biomolecular and Biomedical \\ Research, University College Dublin, Ireland; ${ }^{6}$ Genetics Branch, National Cancer Institute, MD, USA
}

Promoter hypermethylation is central in deregulating gene expression in cancer. Identification of novel methylation targets in specific cancers provides a basis for their use as biomarkers of disease occurrence and progression. We developed an in silico strategy to globally identify potential targets of promoter hypermethylation in prostate cancer by screening for $5^{\prime} \mathrm{CpG}$ islands in 63I genes that were reported as downregulated in prostate cancer. A virtual archive of 338 potential targets of methylation was produced. One candidate, IGFBP3, was selected for investigation, along with glutathione-S-transferase pi (GSTPI), a well-known methylation target in prostate cancer. Methylation of IGFBP3 was detected by quantitative methylation-specific PCR in $49 / 79$ primary prostate adenocarcinoma and 7/14 adjacent preinvasive high-grade prostatic intraepithelial neoplasia, but in only 5/37 benign prostatic hyperplasia $(P<0.000 \mathrm{I})$ and in 0/39 histologically normal adjacent prostate tissue, which implies that methylation of IGFBP3 may be involved in the early stages of prostate cancer development. Hypermethylation of IGFBP3 was only detected in samples that also demonstrated methylation of GSTPI and was also correlated with Gleason score $\geqslant 7(P=0.01)$, indicating that it has potential as a prognostic marker. In addition, pharmacological demethylation induced strong expression of IGFBP3 in LNCaP prostate cancer cells. Our concept of a methylation candidate gene bank was successful in identifying a novel target of frequent hypermethylation in earlystage prostate cancer. Evaluation of further relevant genes could contribute towards a methylation signature of this disease. British Journal of Cancer (2007) 96, I587- 1594. doi: I0.1038/sj.bjc.6603767 www.bjcancer.com

Published online 24 April 2007

(c) 2007 Cancer Research UK

Keywords: methylation; prostate cancer; prostatic intraepithelial neoplasia; insulin-like growth factor binding protein 3; glutathione-Stransferase pi

Prostate cancer is the most common noncutaneous malignancy and a leading cause of cancer-related deaths in men in the Western world (Jemal et al, 2006). Although gland-confined disease is potentially curable, the inevitable emergence of androgen insensitivity in late-stage tumours is ultimately fatal. The molecular events leading to the initiation and development of prostate cancer are not well understood. High-throughout quantitative transcriptomic studies have revealed large numbers of gene expression changes (Dhanasekaran et al, 2001; Ashida et al, 2004), which suggest that a broad and complex network of molecular alterations is involved. In addition to deciphering the identities and functions of these genes, it is important to address the mechanisms that govern their deregulation.

The integral role of epigenetic mechanisms such as promoter hypermethylation in the silencing of tumour suppressor genes has become ever more apparent over the past decade (Herman and Baylin, 2003). Promoter hypermethylation is widespread in prostate cancer; described at early stages of cancer development

\footnotetext{
*Correspondence: Dr AS Perry; E-mail: aperry@tcd.ie

${ }^{7}$ These authors contributed equally to this work.

Received 22 December 2006; revised 28 March 2007; accepted 2 April 2007; published online 24 April 2007
}

in preinvasive high-grade prostatic intraepithelial neoplasia (HGPIN) and has been correlated with clinicopathologic features indicative of a poor prognosis, indicating the potential of gene hypermethylation as a marker of clinically significant disease (Perry et al, 2006).

The most promising methylation biomarker identified to date is glutathione-S-transferase pi (GSTP1), detected in $>90 \%$ of prostate tumours, $>70 \%$ of HGPIN and at significantly lower frequencies and quantitatively much lower levels in normal prostate and benign prostatic hyperplasia (BPH) (Nakayama et al, 2003; Jeronimo et al, 2004). In addition, methylation of GSTP1 is highly specific to prostate cancer, rarely detected in other tumours (Esteller et al, 2001). Glutathione-S-transferase pi is methylated throughout all stages and grades of prostate cancer. Therefore, the efficacy of GSTP1 as a prognostic biomarker of clinically significant prostate cancer would undoubtedly be improved with the discovery of further epigenetic targets in this disease. Identifying novel targets of hypermethylation from transcriptional data repositories may also shed insight into potential pathways of this disease, as well as highlight individual genes relevant to prostate carcinogenesis.

We developed an in silico strategy to globally identify potential targets of promoter hypermethylation in prostate cancer. This approach yielded a database of over 300 potential targets of 
methylation in prostate cancer. Several lines of evidence supported an investigation into the Insulin-like growth factor binding protein 3 (IGFBP3) gene. IGFBP3 is the most abundant IGF-binding protein in the circulation, where it controls the half-life and bioavailability of IGF1 for signal transduction through the IGF1 Receptor (IGF1R), which leads to the activation of growth promoting pathways, including the Ras/Raf/MAP kinase and the phosphotidylinositol-3 kinase pathways (Renehan et al, 2004; Papatsoris et al, 2005). Overexpression of IGFBP3 has dramatic growth-inhibitory and proapoptotic effects in murine prostate tumours and prostate cancer cell lines (Modric et al, 2001; Devi et al, 2002), indicating that IGFBP3 may act as a tumour suppressor in prostate cells. IGFBP3 also exerts a variety of IGFindependent proapoptotic and antiproliferative effects, shown by the finding that IGFBP3 overexpression inhibits the growth of fibroblasts that are IGF1R negative (Oh et al, 1993).

Promoter hypermethylation has been proposed as a mechanism responsible for transcriptional silencing of IGFBP3 in hepatocellular carcinoma (Hanafusa et al, 2002), non-small cell lung carcinoma (Chang et al, 2002) and very recently in cancers of the bladder and ovary (Christoph et al, 2006; Wiley et al, 2006). In this study, we performed an extensive analysis of the methylation pattern of IGFBP3 in benign, preinvasive and cancerous prostate tissues. Concordance between IGFBP3 and GSTP1 methylation was investigated. Statistical analysis was performed to investigate a correlation between methylation and clinical and pathologic parameters. In addition, prostate cancer cell lines were employed to test whether promoter hypermethylation affects IGFBP3 gene expression.

\section{MATERIALS AND METHODS}

\section{In silico mining to identify novel targets of methylation in prostate cancer}

We developed an in silico approach to identify potential targets of promoter hypermethylation in prostate cancer. The principle behind this concept was that promoter hypermethylation leads to a reduction in gene expression at the transcriptional level. Two freely available web-based transcriptome databases were employed to identify genes downregulated in prostate cancer compared with normal prostate. Nine queries were performed on the Gene Expression Atlas (http://expression.gnf.org) to retrieve genes that were both expressed in normal prostate $>1$ times - and in prostate cancer $<0.5-1$ times - the median expression across 46 human tissues (Su et al, 2001). The Digital Differential Display (http:// www.ncbi.nlm.nih.gov/UniGene/ddd) was used to report on significant differences in gene expression between four different tissue pools, created from libraries of ESTs from normal prostate, primary and metastatic prostate cancer and PIN (Wheeler et al, 2001). The data from four independent, published microarray studies that quantified gene expression at different stages of prostate cancer were also examined (Bubendorf et al, 1999; Chetcuti et al, 2001; Dhanasekaran et al, 2001; Ashida et al, 2004).

Each gene was screened for the presence of a $5^{\prime} \mathrm{CpG}$ island using the UCSC Human Genome Browser (http://genome.ucsc.edu/) to positively filter for genes that could be susceptible to promoter hypermethylation (Kent et al, 2002). Successful targets were organised by putative function using GeneCards (http://genecards. org) (Rebhan et al, 1997). CpG islands were characterised for promoter sequences using Promoter Scan (http://thr.cit.nih.gov/ molbio/proscan/) (Prestridge, 1995).

\section{Clinical sample collection}

Prostate tumours and histologically normal adjacent tissue from 40 patients with primary disease, treated by radical prostatectomy at The Adelaide and Meath Hospital (AMNCH), Dublin, Ireland, were obtained retrospectively. Histologically normal adjacent tissue was tissue not involved by prostate cancer, HGPIN or BPH. High-grade prostatic intraepithelial neoplasia lesions were obtained from 14 cases. For control purposes, BPH lesions from 37 men without prostate cancer that underwent transurethral resection (TURP) of the prostate were also collected. Additionally, a further 39 primary tumours were obtained from the Florida Hospital Cancer Institute, USA (FHCI) as part of a collaboration with the National Cancer Institute (NCI), USA. The Gleason score, TNM classification (Fleming et al, 1997), PSA level and age at diagnosis were obtained from the relevant clinical records. This study was approved by the AMNCH and St James's Hospital Ethics committee. The FHCI specimens were approved for research purposes by the NCI Office of Human Subjects Research.

Histological slides from the formalin-fixed, paraffin-embedded (FFPE) surgical specimens were reviewed by a pathologist to identify areas of histologically normal prostate, prostate cancer, HGPIN and BPH. A series of $5 \mu \mathrm{m}$ sections were cut from the FFPE blocks. The first tissue section from each block was hematoxylin and eosin stained and compared with the pathologically evaluated slide, to ensure a consistent percentage of target cells. The target cell populations were the glandular epithelial cells. Adjacent sections were deparaffinised in xylene and rehydrated in decreasing concentrations of ethanol. Tissue was scraped from within the pathologically marked areas using a sterile blade and DNA was extracted using the QIAamp DNA micro kit (Qiagen, Crawley, UK).

\section{Cell culture and drug treatment}

Human prostate cancer cell lines LNCaP, DU145 and PC-3 and normal prostate cell line PWR-1E were propagated under standard cell culture conditions. The effect of DNA methylation on IGFBP3 expression was measured in LNCaP cells by treatment with the demethylating agent 5 -azacytidine. LNCaP cells $\left(2 \times 10^{6}\right)$ were seeded in $75 \mathrm{~cm}^{3}$ culture flasks. Twenty-four hours after plating, cells were treated with $2 \mu \mathrm{m}$ 5-azacytidine (Sigma, Dublin, Ireland) and the medium was changed after $24 \mathrm{~h}$. After 2 days of treatment, cells were harvested. DNA was isolated from cell lines by use of a QIAamp DNA mini kit (Qiagen, UK) and total RNA was extracted using the Trizol method (Invitrogen, Paisley, UK).

\section{Bisulfite modification and quantitative methylation specific PCR}

Sodium bisulphite modification of genomic DNA converts unmethylated (but not methylated) cytosines to uracils. Bisulfite modification of approximately $20 \mathrm{ng}$ of DNA isolated from tissue samples and up to $1 \mu \mathrm{g}$ of cell line DNA, was performed with the EZ DNA methylation kit (Zymo Research, Orange, CA, USA). Modified DNA was eluted into a final volume of $50 \mu \mathrm{l} 1 \times$ Tris EDTA (Sigma, Ireland).

Methylation was evaluated by real time quantitative methylation specific PCR (QMSP) as described by Eads et al (2000). Parallel TaqMan PCR reactions were performed on every sample with oligonucleotides targeted to an (i) endogenous control gene $(\beta$-actin) for unbiased amplification of bisulphite modified DNA and (ii) target genes (GSTP1 and IGFBP3) for amplification of bisulphite modified fully methylated molecules (Table 1). A quantity of product was obtained for each reaction by interpolating from a standard curve, constructed with 10-fold serial dilutions of bisulfite modified, universal methylated DNA (Chemicon International, Temecula, CA, USA). The level of methylated target in each sample was then determined by a relative methylation score (RMS), by applying the formula: (target quantity/ $\beta$-actin quantity) $\times 1000$.

All assays were performed in duplicate on a 7900 HT Sequence Detection System (Perkin-Elmer, Foster City, CA, USA), in a final 
Table I QMSP primer and probe sequences

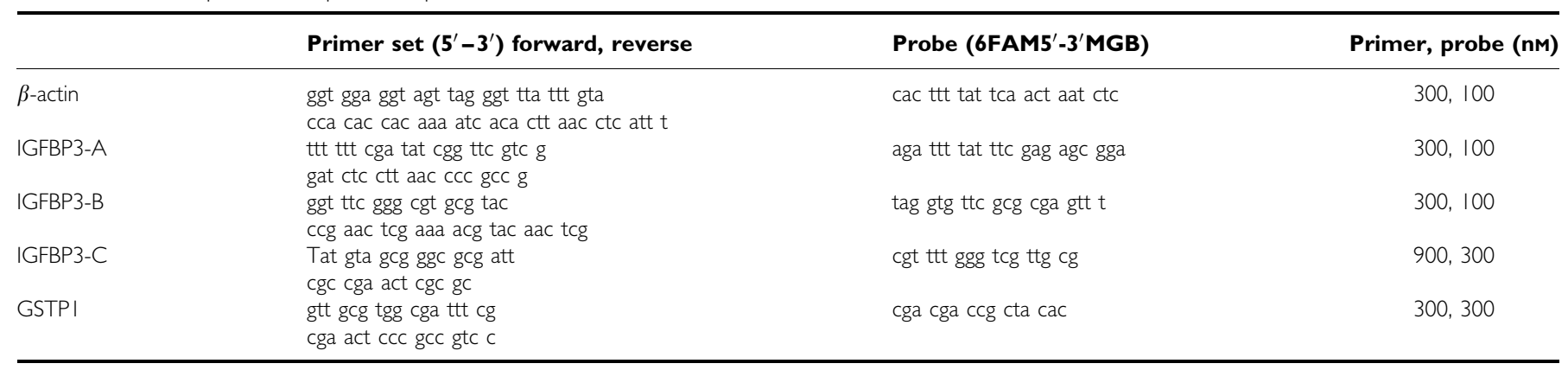

volume of $10 \mu \mathrm{l}$, which consisted of $2 \mu \mathrm{l}$ bisulfite-modified DNA, primers (MWG Biotech, Ebersberg, Germany), fluorescent labelled probe (Applied Biosystems, Warrington, UK) and $5 \times$ TaqMan Universal PCR master mix, no AmpErase Uracil N-Glycosylase (Roche, Branchburg, NJ, USA), under standard TaqMan real-time PCR cycling conditions. Samples were considered positively amplified when a comparative threshold cycle $\left(C_{\mathrm{T}}\right)<50$ was detected in both duplicates, with $<1 C_{\mathrm{T}}$ variance between duplicates.

Methylation of IGFBP3 was investigated by three QMSP assays designed to evaluate $\mathrm{CPG}$ dinucleotides at different locations (promoter and coding sequence) within the promoter $\mathrm{CpG}$ island: IGFBP3-A: -406 to -328 , IGFBP3-B: -217 to -150 and IGFBP3-C: +132 to +228 . Altogether, the primer and probe sets covered $30 /$ $139 \mathrm{CpG}$ sites throughout the region, spanning more than $600 \mathrm{bp}$ of the island.

\section{Quantitative reverse transcription PCR}

cDNA was synthesised from $1 \mu \mathrm{g}$ RNA using M-MLV reverse transcriptase and random hexamers (Invitrogen, UK) at $37^{\circ} \mathrm{C}$ for $1 \mathrm{~h}$ in a total volume of $30 \mu \mathrm{l}$. A primer and probe set was targeted to exons 2 and 3 of the IGFBP3 gene, to avoid amplification of DNA, forward primer $5^{\prime}$-agtccaagcgggagacagaa- $3^{\prime}$, reverse primer $5^{\prime}$-caggtgattcagtgtgtcttcca- $3^{\prime}$, probe $6 \mathrm{FAM} 5^{\prime}$-ccctgccgtaga gaa- $3^{\prime}$ MGB. cDNA (100 ng) was used as a template for real-time TaqMan reactions, as described above. The levels of IGFBP3 expression in cell lines were calculated as a fold change from a calibrator sample (normal prostate cell line PWR-1E) using the Comparative $C_{\mathrm{T}}$ method by applying the formula $2^{-\Delta \Delta C_{\mathrm{T}}}$ (Livak and Schmittgen, 2001). The amount of IGFBP3 in each sample was normalised to an endogenous reference gene, using a $\beta$-actin pre-developed assay (Applied Biosystems, UK).

\section{Statistical analysis}

Statistical analysis was performed using MINITAB (version 1.4). Differences in frequencies of methylation between histologically normal, BPH, HGPIN and prostate cancer were assessed using Fisher's exact Test. Differences in methylation levels of genes were compared by examining the RMSs between samples using the Kruskal-Wallis one-way ANOVA test and the Wilcoxon-matched pairs test. An Unpaired $t$-test was used to calculate the differences in age and PSA between patient groups. For all of the tests, significance was ascribed at $P<0.05$.

\section{RESULTS}

\section{Identification of potential targets of methylation in prostate cancer}

A list of 631 (known and unknown) genes that are downregulated in prostate cancer was generated through our in silico strategy.
Of these genes, 355 (56.26\%) possessed a CpG island within $5 \mathrm{~kb}$ $+I-$ of their transcriptional start site (Supplementary Table 1). $16 / 355$ genes were commonly identified as downregulated in more than one study (ATF3, CAV2, CCND2, CTSB, CYP1B1, EPB72, GSTM1, ID2, IGFBP3, MGFE8, NET1A, OAT, RPS19, SGK, SHB and ZFP36). The appearance of known targets of methylation, CCND2, $C D 44, E C A D$ and GADD45A, demonstrated the success of this technique in identifying (potential) targets of methylation in prostate cancer. We selected IGFBP3 as our first target to evaluate.

\section{Clinical characteristics}

Methylation was investigated in tissue samples of prostate cancer, histologically normal prostate, HGPIN and BPH. As expected, PSA levels were higher in patients with prostate cancer $(9.74 \mathrm{ng} \mathrm{ml})$ than with $\mathrm{BPH}\left(6.39 \mathrm{ng} \mathrm{ml}^{-1}\right)(P=0.018,95 \% \mathrm{CI}=1.15,3.72)$, although there was considerable overlap in the range of measures. Notably, the mean PSA of the BPH patients was above $4.0 \mathrm{ng} \mathrm{ml}^{-1}$, the widely accepted upper limit of normal. The difference in mean age between the prostate cancer (61.67 years) and BPH (75.41 years) groups was statistically significant $(P<0.0001,95 \%$ $\mathrm{CI}=10.50,16.97)$.

\section{IGFBP3 methylation in prostate tissues}

The characterised promoter CpG island of IGFBP3 is shown in Figure 1. IGFBP3 methylation was detected in $49 / 79(62.03 \%)$ of the prostate cancer samples at sequence A, 15/79 (19.23\%) at sequence $B$ and $0 / 79(0 \%)$ at sequence $C$ (Figure 2).

IGFBP3 was completely unmethylated in the histologically normal prostate samples, except for two cases that contained methylated DNA in the IGFBP3-C region. In the BPH samples, the frequencies of methylation were very low: IGFBP3-A, 5/37 (13.51\%); IGFBP3-B, 2/37 (5.41\%) and IGFBP3-C 0/37 (0\%). In total, $7 / 37(18.9 \%)$ of the hyperplastic prostates showed methylation of $I G F B P 3$, a frequency significantly less than detected in the tumours $(P<0.0001)$. There was no significant difference in the methylation frequency between the histologically normal and BPH groups.

HGPIN lesions were obtained from 14/79 patients with prostate cancer. IGFBP3 methylation was only detected in HGPIN samples from patients whose adjacent tumour was also methylated. IGFBP3-A methylation was found in 7/14 (50\%) cases, IGFBP3-B in $1 / 14(7.14 \%)$ cases and IGFBP3-C in $0 / 14$ cases. The frequency of IGFBP3 methylation in HGPIN was not statistically different from tumour $(P=0.383)$.

\section{Methylation of GSTP1 and IGFBP3}

Hypermethylation of GSTP1 was identified in 75/79 (94.94\%) tumours, $10 / 14$ (71.43\%) HGPIN, 5/39 (12.82\%) histologically normal prostate samples and $4 / 37(10.81 \%)$ BPH. Statistically significant differences were found for the methylation status of 
CgcctgccaaCGgaattaaattttagaaagctccaCGaggtacacaCGaatgCGgagCGctgtatgccagtttcccCGacacCGgctCGgCGcagggaga cctcaccCCGagagCGgaaggggtaagggGGgCGgggtcaaggagatCGggggtgctgagttggccaggagtgactggggtgacCGggggtgctgaggtg $A-P R \quad U C E .2 \quad-1253$ A-R 553

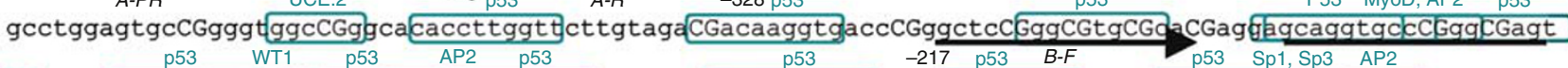

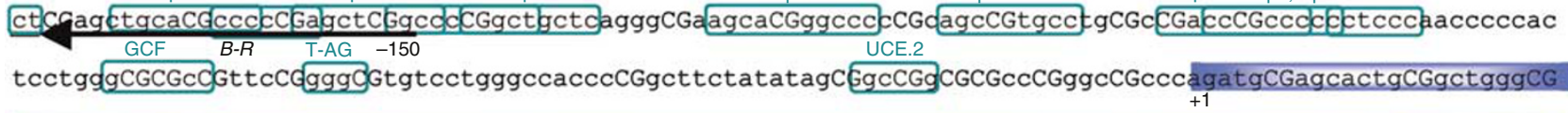

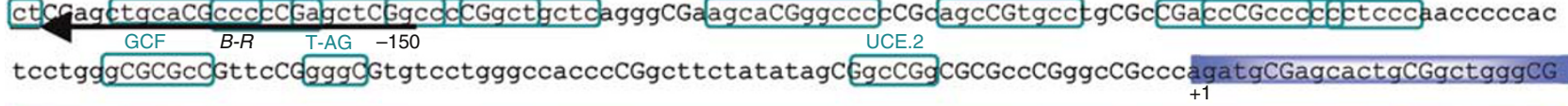

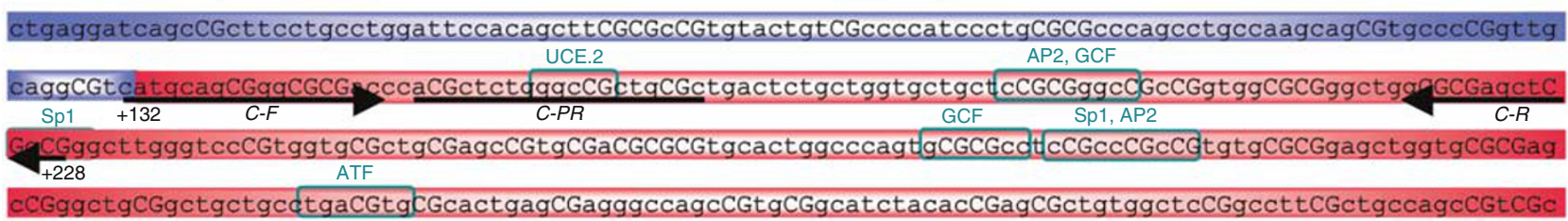

cCGaCGaggCGCGacCGctgcaggCGctgctggaCGgcCGCGggctctgCGtcaaCGctagtgcCGtcagcCGcctgCGCGcctacctgctgccagCGcC

GccagctccaggtgagcCGccCGCGccaggtgCGctgCGtgcagcaccCGgCGcactggCGcCGaagggcetgggggttgctgggtgcCGctgCGggaga

ctcCGcttttcttctcactggagataatatgtggggaaactgaaggCGctcCGggaaaggtgaaggCGgtCGcCGgagggaccctccccagcCGgccctc

tacttgctC

Figure I Genomic sequence analysis of IGFBP3 (GenBank accession number M35878). The 1.2 kb IGFBP3 CpG island begins 476 bp upstream of the 5' untranslated region (shown in blue), extends through the first exon (shown in red) and terminates 199 bp into the first intron. Putative transcription factor binding sites that contain CPG sites are indicated. Primer and probe sequences for QMSP assays (A, B and $\mathbf{C})$ are shown with black arrows and lines, respectively.

GSTP1 between prostate cancer and both BPH and histologically normal adjacent tissues $(P<0.0001)$. In all 49 tumours and $6 / 7$ HGPIN that showed IGFBP3 methylation, methylation of GSTP1 was also detected. Methylation of IGFBP3 was only detected in those samples (both tumour and the majority of HGPIN) that demonstrated GSTP1 methylation. Notably, methylation of both genes was not detected in any of the histologically normal or BPH samples.

\section{Methylation levels in prostate tissues}

In addition to determining the frequencies of IGFBP3 and GSTP1 methylation, the levels of methylation were compared between the different sample types (Figure 3). Although there was a significant difference between the methylation levels of GSTP1 and IGFBP3 within tumour samples $(P<0.0001 ; 95 \% \mathrm{CI}=88.17,174.22)$, the median RMS of both genes was significantly higher in tumours than in histologically normal adjacent prostate or BPH $(P<0.0001)$.

\section{Promoter hypermethylation and clinicopathologic correlations}

Results for correlations of the methylation status of IGFBP3 and GSTP1 with clinicopathologic factors (Gleason score, TNM classification and PSA level) are shown in Table 2. Methylation of GSTP1 was not significantly associated with any pathological parameters. Methylation of IGFBP3 was detected in significantly more tumours with Gleason score $\geqslant 7$, than $\leqslant 6(P=0.01)$, but was not significantly correlated with TNM classification or PSA level.

\section{IGFBP3 methylation and gene silencing in prostate cancer cell lines}

The methylation pattern of IGFBP3 was examined in a panel of prostate cancer cell lines (Figure 4). Only the LNCaP cell line was found to possess methylated IGFBP3 alleles by amplification with the A and B primer sets. The demethylation of IGFBP3 by 5azacytidine in this cell line was confirmed by QMSP. QRT-PCR revealed that the three cancer cell lines expressed IGFBP3 relative to the normal cell line PWR-1E, however, pharmacological demethylation of $\mathrm{LNCaP}$ increased the expression of IGFBP3 several hundred-fold.

\section{DISCUSSION}

Recognition of the importance of promoter hypermethylation in human cancer has fostered a growing effort to screen the cancer genome to identify methylated loci. In this study, we describe a novel in silico approach to identify potential targets of methylation in prostate cancer. We employed transcriptomic databases and microarray experiments that provided detailed data summaries and focused on expression changes in early-stage disease (Chetcuti et al, 2001; Ashida et al, 2004) and in the progression to metastatic disease (Bubendorf et al, 1999; Dhanasekaran et al, 2001). Interestingly, a common finding between these studies was that downregulation rather than upregulation, accounted for the majority of differentially expressed genes in prostate cancer. This suggests that the full force of transcriptional silencing mechanisms in prostate cancer may not yet be fully recognised.

Approximately half of the genes examined contained a $5^{\prime} \mathrm{CpG}$ island, which was consistent with previous reports (Takai and Jones, 2002; Wang and Leung, 2004). To select the most interesting gene(s) for methylation analysis from over 300 candidates required further validation, beyond the identification of a promoter $\mathrm{CpG}$ island. Furthermore, we recognised that there are substantial challenges in the interpretation of data obtained from large-scale gene expression arrays. For example, which of the hundreds of differentially expressed genes are important primary events and which are downstream or secondary events? To maximise the likelihood of choosing the most relevant genes for analysis, literature review was performed on all of the candidates. This led to the selection of the IGFBP3 gene, whose methylation status has not been previously reported on in prostate cancer.

A detailed map of the IGFBP3 promoter CpG island facilitated QMSP assay design around functionally relevant regions of the island. Using three QMSP assays, we found tumour-related differential methylation of the IGFBP3 promoter CpG island in prostate cancer. Methylation was exclusive to the non-coding part 

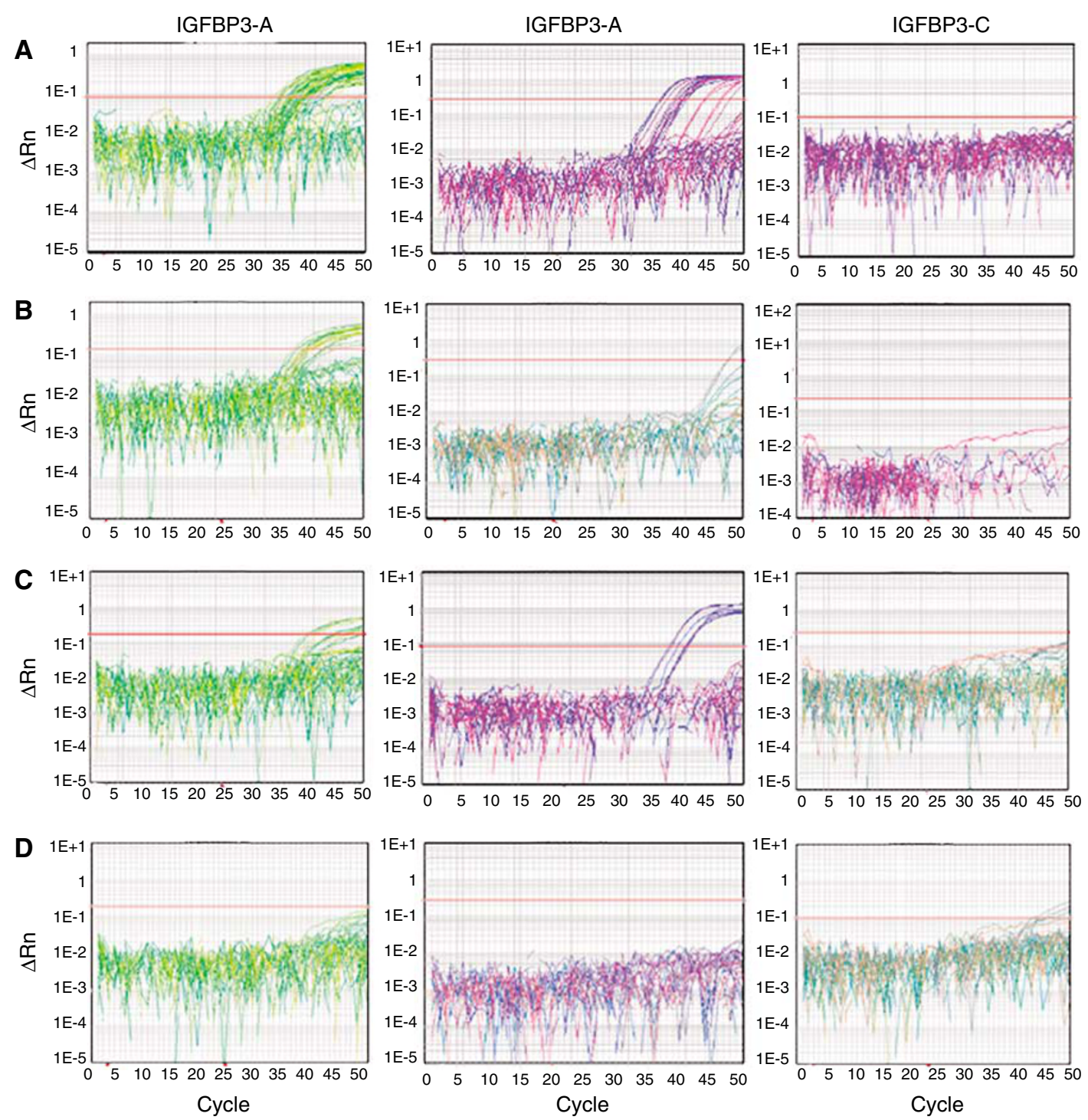

Figure 2 Differential methylation of IGFBP3 in prostate cancer. QMSP amplification plots in (A) prostate cancer, (B) HGPIN, (C) BPH and (D) histologically normal adjacent prostate samples. Methylation was only detected in two of the morphologically normal samples at IGFBP3-C at cycle number 43 , indicating very low levels of methylation in these samples.

of the island. The high frequency (62\%) observed at the $5^{\prime}$ end of the island (assay-A) decreased to $19 \%$ around the MyoD, AP-2, p53 and WT-1 transcription factor binding sites (assay-B) and diminished to zero at the start of the first exon (assay-C). Of the 15 tumours methylated at assay-B, all but one were also methylated at the upstream part of the island, which supports the theory that de novo hypermethylation may gradually propagate through an island during neoplastic progression, initiating from the outer flanks of the CpG island (Mummaneni et al, 1995; Graff et al, 1997). An interesting finding was that the majority of prostate cancers were unmethylated around a methylation hotspot (corresponding to the $\mathrm{p}-53, \mathrm{AP}-2$ and Sp-1/Sp3 binding sites), reported in both non-small cell lung carcinoma (61\%) and hepatocellular carcinoma (33\%) (Chang et al, 2002; Hanafusa et al, 2002). These results clearly show that methylation is not uniformly spread throughout the IGFBP3 $\mathrm{CpG}$ island and therefore, the choice of $\mathrm{CpG}$ sites for investigation is an important consideration.

To address whether IGFBP3 methylation is involved in the early stages of disease initiation, we examined the relationship between methylation of IGFBP3 in prostate cancer and HGPIN isolated from the same prostatectomy samples. Methylation was only detected in those HGPIN lesions, whose adjacent tumour sample from the same patient was also methylated. This implies that hypermethylation of IGFBP3 occurs as an early event in prostate carcinogenesis and supports a clonal relation between the tumour and HGPIN lesion. We also found methylation of GSTP1 in a high frequency of HGPIN foci, consistent with previous reports (Nakayama et al, 2003; Kang et al, 2004).

Although the frequency and levels of GSTP1 methylation were significantly higher than IGFBP3 methylation, an unexpected finding was the concordance between methylation of both genes across all tumours and all but one HGPIN. The high prevalence of GSTP1 hypermethylation in prostate cancer, HGPIN and in some proliferative inflammatory atrophy lesions indicates that it most likely precedes many other molecular aberrations in prostate carcinogenesis (Nakayama et al, 2003). Associated loss of GSTP1 activity and its protection from electrophilic and oxidative DNA damage would render cells susceptible to further transformations. Promoter hypermethylation of IGFBP3 likely occurs as a subsequent epigenomic 'hit' in the multi-step process of cancer development.

Low levels of methylation were detected for both IGFBP3 and GSTP1 in a small percentage of benign prostate samples. 

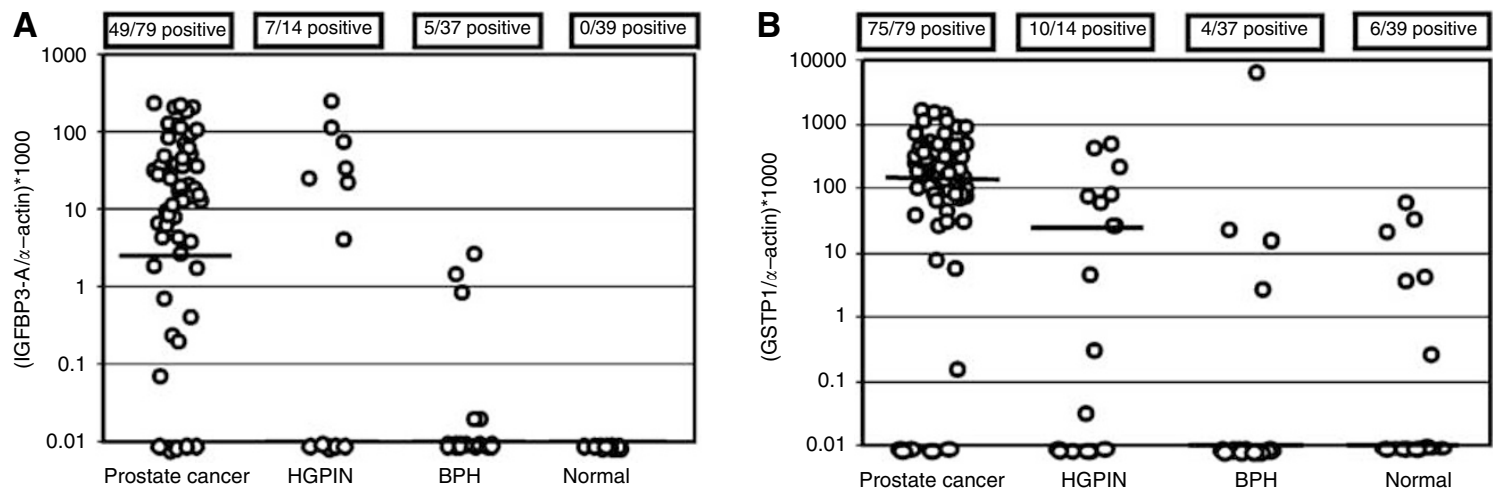

Figure 3 Distribution of (A) IGFBP3-A and (B) GSTPI methylation levels in prostate cancer, HGPIN, normal prostate and BPH. The median RMS is indicated by a horizontal line. Values diagrammed at 0.01 are zero values, which could not be plotted correctly on a log scale.

Table 2 Frequency of IGFBP3 and GSTPI methylation in histologically normal and cancerous prostate tissue, HGPIN and BPH, and relationship to clinicopathologic factors

\begin{tabular}{|c|c|c|c|c|}
\hline & $\begin{array}{c}\text { IGFBP3-A } \\
n(\%)\end{array}$ & $\begin{array}{c}\text { IGFBP3-B } \\
n \text { (\%) }\end{array}$ & $\begin{array}{c}\text { IGFBP3-C } \\
n(\%)\end{array}$ & $\begin{array}{c}\text { GSTPI } \\
\text { n (\%) }\end{array}$ \\
\hline Normal prostate & 0/39 (0) & 0/39 (0) & 2/39 (5.3) & $5 / 39(12.82)$ \\
\hline $\mathrm{BPH}$ & $5 / 37(|3.5|)$ & 2/37 (5.4I) & 0/37 (0) & $4 / 37(10.81)$ \\
\hline HGPIN & $7 / 14(50)$ & $1 / 14(7.14)$ & $0 / 14(0)$ & $10 / 14(71.43)$ \\
\hline Prostate cancer & $49 / 79(62.03)$ & I5/79 (19.23) & $0 / 79(0)$ & $75 / 79(94.94)$ \\
\hline \multicolumn{5}{|l|}{ Gleason score } \\
\hline$\leqslant 6$ & 17/37 (45.95) & $4 / 37(|0.8|)$ & & $35 / 37$ (94.59) \\
\hline$\geqslant 7$ & $32 / 42(76.19)$ & I I/42 (26.19) & & $40 / 42(95.24)$ \\
\hline$P$-value & 0.01 & 0.09 & & i \\
\hline \multicolumn{5}{|l|}{ TNM classification } \\
\hline pT2 & $35 / 55(63.64)$ & I I/55 (20) & & $51 / 55(94.44)$ \\
\hline pT3, pT4 & $12 / 20(60)$ & $3 / 20(15)$ & & $20 / 20(100)$ \\
\hline$P$-value & 0.8 & 0.8 & & $i$ \\
\hline \multicolumn{5}{|l|}{ PSA (ng ml) } \\
\hline$<4$ & $5 / 9(55.56)$ & $1 / 9(|1| 1)$. & & 8/9 (88.89) \\
\hline $4-10$ & $27 / 43(62.79)$ & $6 / 43(13.95)$ & & $42 / 43(97.67)$ \\
\hline$>10$ & $13 / 22(59.1)$ & $6 / 22(27.27)$ & & 20/22 (90.91) \\
\hline$P$-value & 0.9 & 0.37 & & 0.38 \\
\hline
\end{tabular}

Consistent with our results, other studies have also described quantitatively much less promoter methylation of GSTP1 in BPH and histologically normal tissue (from tumour containing prostates) than in cancer specimens (Jeronimo et al, 2001; Yamanaka et al, 2003). Although every effort was taken to ensure that tissue was only procured from within pathologically identified areas, we cannot rule out the possibility that minute amounts of occult carcinoma or HGPIN may have been present in some of the benign specimens. However, even studies that employed laser capture microdissection to isolate pure populations of cells have reported low levels of hypermethylation in morphologically normal prostate samples (Henrique et al, 2006). An alternative explanation is that certain molecular aberrations such as promoter hypermethylation may precede morphological changes, initially affecting a small subset of benign epithelial cells. In support of this hypothesis, none of the benign samples demonstrated any evidence of methylation spreading, that is, they were not that amplifiable with primer and probe sets IGFBP3-A and IGFBP3-B.

Reduced expression of IGFBP3 in the prostate epithelium is correlated with cancer progression from HGPIN to localised cancer and androgen-independent disease (Thrasher et al, 1996; Hampel et al, 1998; Nickerson et al, 2001). Rising PSA levels during the course of prostate cancer progression may facilitate tumorigenesis
A

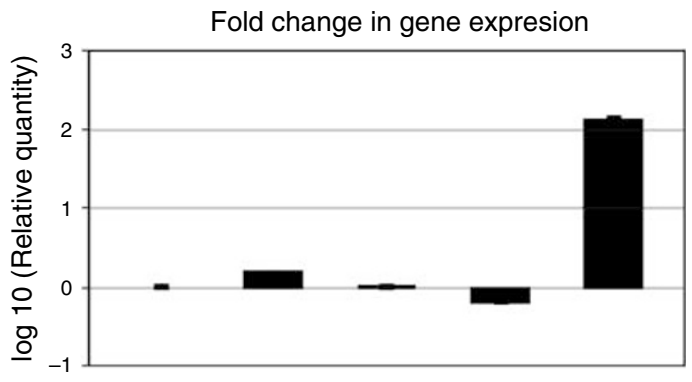

\begin{tabular}{|c|c|c|c|c|c|}
\hline $\begin{array}{l}\text { Methylation } \\
\text { status }\end{array}$ & $\underset{⿱ 亠 乂}{\stackrel{\varpi}{\check{\alpha}}}$ & ర్ & $\begin{array}{l}\frac{10}{5} \\
\frac{5}{0}\end{array}$ & 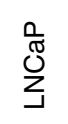 & 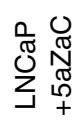 \\
\hline IGFBP3-A & --- & --- & --- & ++- & +-- \\
\hline IGFBP3-B & --- & --- & --- & +-- & +-- \\
\hline IGFBP3-C & --- & --- & --- & --- & --- \\
\hline
\end{tabular}

Figure 4 IGFBP3 methylation and expression analyses in prostate cancer cell lines. (A) The fold change in mRNA expression of IGFBP3 in prostate cancer cell lines relative to normal prostate cell line PWRIE. QRT-PCR showed that all cell lines expressed IGFBP3 mRNA but the LNCaP cell line showed $\sim 3$-fold reduction in expression. This was reversed upon treatment with demethylating drug 5-azacytidine. (B) The relative level of IGFBP3 methylation in cell lines was examined by QMSP and is displayed by +++ : 1000, $++-:$ 999-500, $-+:$ 499-I and -: 0 .

by digesting IGFBP3 and releasing free IGF-1 into the prostate microenvironment (Cohen et al, 1992). However, this posttranslational proteolysis cannot account for reduced IGFBP3 mRNA levels (Ashida et al, 2004; Thelen et al, 2004). Examination of IGFBP3 expression in vitro, revealed that all of the prostate cancer cell lines expressed IGFBP3, including LNCaP cells whose methylation pattern was representative of the majority of prostate tumours analysed (heavily methylated at IGFBP3-A, remarkably fewer CpGs methylated at IGFBP3-B and unmethylated at IGFBP3$C)$. However, 5-azacytidine treatment of $\mathrm{LNCaP}$ reversed the hypermethylation pattern of IGFBP3 and resulted in a significant induction in expression. Although these findings could indicate monoallelic methylation in $\mathrm{LNCaP}$, previous reports would suggest that expression is maintained because methylation is not affecting core promoter sequences (Chang et al, 2004; Hanafusa et al, 2005). Whether methylation spreading throughout the island in approximately $20 \%$ of prostate cancers correlates with a reduction in IGFBP3 expression, warrants further investigation.

With a shift toward earlier stages at diagnosis because of PSA screening, it is becoming increasingly important to understand the aetiology of prostate cancer recurrence and progression. 
Identifying methylation events early in carcinogenesis that are correlated with potentially aggressive tumours could identify those patients more likely to recur and avoid the over-treatment of those indolent tumours that may otherwise never require therapy. In support of previous studies, our data show methylation of GSTP1 is highly prevalent across all grades and stages of disease (Jeronimo et al, 2001; Yegnasubramanian et al, 2004). However, methylation of IGFBP3 was associated with advanced tumour grade. Increased density of methylation within a CpG island has been associated with more advanced stages of tumours (Nosaka et al, 2000). Although we did not detect a statistically significant relationship between tumour samples amplified by both primer sets IGFBP3-A and IGFBP3-B and Gleason score $(P=0.09)$, this may be attributable in part to the small sample size. Non-small cell lung carcinoma patients with methylation of IGFBP3 had significantly poorer overall survival probability compared with those without methylation (Chang et al, 2002). In addition, methylation levels of IGFBP3 have been associated with tumour recurrence in transitional cell carcinoma of the bladder (Christoph et al, 2006). The results from this study indicate that such an investigation is warranted in prostate cancer.

In summary, our concept of in silico data mining was successful in identifying a novel target of frequent methylation in prostate cancer and HGPIN. Our findings also emphasise the importance of thorough investigations into methylation patterns in order to gain an insight into the dynamics of de novo hypermethylation and its relationship to transcriptional silencing. Although GSTP1 is widely recognised as an excellent biomarker of prostate cancer, used alone it has limitations. The inherent clinical and genetic heterogenic nature of prostate cancer suggests that profiling the cumulative methylation of multiple genes would serve to better distinguish benign from malignant tissues and would provide a more powerful approach in the early detection of prostate cancer and in identifying those men who should be targeted for more aggressive therapy, than characterising the status of only one gene marker. We are screening further potential targets of methylation that we have identified to contribute towards a methylation fingerprint of this disease.

\section{ACKNOWLEDGEMENTS}

This work was supported by the Irish Research Council for Science, Engineering and Technology and the Irish Cancer Society. We thank Professor Ralph deVere White, Dr Lukasz Huminecki and Dr Andrew Lloyd for their advice on this paper.

Supplementary Information accompanies the paper on British Journal of Cancer website (http://www.nature.com/bjc)

\section{REFERENCES}

Ashida S, Nakagawa H, Katagiri T, Furihata M, Iiizumi M, Anazawa Y, Tsunoda T, Takata R, Kasahara K, Miki T, Fujioka T, Shuin T, Nakamura Y (2004) Molecular features of the transition from prostatic intraepithelial neoplasia (PIN) to prostate cancer: genome-wide gene-expression profiles of prostate cancers and PINs. Cancer Res 64: $5963-5972$

Bubendorf L, Kolmer M, Kononen J, Koivisto P, Mousses S, Chen Y, Mahlamaki E, Schraml P, Moch H, Willi N, Elkahloun AG, Pretlow TG, Gasser TC, Mihatsch MJ, Sauter G, Kallioniemi OP (1999) Hormone therapy failure in human prostate cancer: analysis by complementary DNA and tissue microarrays. J Natl Cancer Inst 91: 1758-1764

Chang YS, Wang L, Liu D, Mao L, Hong WK, Khuri FR, Lee HY (2002) Correlation between insulin-like growth factor-binding protein-3 promoter methylation and prognosis of patients with stage I non-small cell lung cancer. Clin Cancer Res 8: $3669-3675$

Chang YS, Wang L, Suh YA, Mao L, Karpen SJ, Khuri FR, Hong WK, Lee HY (2004) Mechanisms underlying lack of insulin-like growth factorbinding protein-3 expression in non-small-cell lung cancer. Oncogene 23: $6569-6580$

Chetcuti A, Margan S, Mann S, Russell P, Handelsman D, Rogers J, Dong Q (2001) Identification of differentially expressed genes in organ-confined prostate cancer by gene expression array. Prostate 47: 132-140

Christoph F, Weikert S, Kempkensteffen C, Krause H, Schostak M, Miller K, Schrader M (2006) Regularly methylated novel pro-apoptotic genes associated with recurrence in transitional cell carcinoma of the bladder. Int J Cancer 119: 1396-1402

Cohen P, Graves HC, Peehl DM, Kamarei M, Giudice LC, Rosenfeld RG (1992) Prostate-specific antigen (PSA) is an insulin-like growth factor binding protein-3 protease found in seminal plasma. J Clin Endocrinol Metab 75: $1046-1053$

Devi GR, Sprenger CC, Plymate SR, Rosenfeld RG (2002) Insulin-like growth factor binding protein-3 induces early apoptosis in malignant prostate cancer cells and inhibits tumor formation in vivo. Prostate 51: $141-152$

Dhanasekaran SM, Barrette TR, Ghosh D, Shah R, Varambally S, Kurachi K, Pienta KJ, Rubin MA, Chinnaiyan AM (2001) Delineation of prognostic biomarkers in prostate cancer. Nature 412: $822-826$

Eads CA, Danenberg KD, Kawakami K, Saltz LB, Blake C, Shibata D, Danenberg PV, Laird PW (2000) MethyLight: a high-throughput assay to measure DNA methylation. Nucleic Acids Res 28: E32

Esteller M, Corn PG, Baylin SB, Herman JG (2001) A gene hypermethylation profile of human cancer. Cancer Res 61: 3225-3229
Fleming ID, Cooper JS, Henson DE (1997) American Joint Committee on Cancer Staging Manual. Lippincott: Philadelphia

Graff JR, Herman JG, Myohanen S, Baylin SB, Vertino PM (1997) Mapping patterns of $\mathrm{CpG}$ island methylation in normal and neoplastic cells implicates both upstream and downstream regions in de novo methylation. J Biol Chem 272: 22322-22329

Hampel OZ, Kattan MW, Yang G, Haidacher SJ, Saleh GY, Thompson TC, Wheeler TM, Marcelli M (1998) Quantitative immunohistochemical analysis of insulin-like growth factor binding protein-3 in human prostatic adenocarcinoma: a prognostic study. J Urol 159: 2220-2225

Hanafusa T, Shinji T, Shiraha H, Nouso K, Iwasaki Y, Yumoto E, Ono T, Koide N (2005) Functional promoter upstream p53 regulatory sequence of IGFBP3 that is silenced by tumor specific methylation. BMC Cancer 5: 9

Hanafusa T, Yumoto Y, Nouso K, Nakatsukasa H, Onishi T, Fujikawa T, Taniyama M, Nakamura S, Uemura M, Takuma Y, Yumoto E, Higashi T, Tsuji T (2002) Reduced expression of insulin-like growth factor binding protein-3 and its promoter hypermethylation in human hepatocellular carcinoma. Cancer Lett 176: 149-158

Henrique R, Jeronimo C, Teixeira MR, Hoque MO, Carvalho AL, Pais I, Ribeiro FR, Oliveira J, Lopes C, Sidransky D (2006) Epigenetic heterogeneity of high-grade prostatic intraepithelial neoplasia: clues for clonal progression in prostate carcinogenesis. Mol Cancer Res 4: $1-8$

Herman JG, Baylin SB (2003) Gene silencing in cancer in association with promoter hypermethylation. N Engl J Med 349: 2042-2054

Jemal A, Siegel R, Ward E, Murray T, Xu J, Smigal C, Thun MJ (2006) Cancer statistics, 2006. CA Cancer J Clin 56: 106-130

Jeronimo C, Henrique R, Hoque MO, Mambo E, Ribeiro FR, Varzim G, Oliveira J, Teixeira MR, Lopes C, Sidransky D (2004) A quantitative promoter methylation profile of prostate cancer. Clin Cancer Res 10: $8472-8478$

Jeronimo C, Usadel H, Henrique R, Oliveira J, Lopes C, Nelson WG, Sidransky D (2001) Quantitation of GSTP1 methylation in nonneoplastic prostatic tissue and organ-confined prostate adenocarcinoma. J Natl Cancer Inst 93: 1747-1752

Kang GH, Lee S, Lee HJ, Hwang KS (2004) Aberrant CpG island hypermethylation of multiple genes in prostate cancer and prostatic intraepithelial neoplasia. J Pathol 202: 233-240

Kent WJ, Sugnet CW, Furey TS, Roskin KM, Pringle TH, Zahler AM, Haussler D (2002) The human genome browser at UCSC. Genome Res 12: $996-1006$ 
Livak KJ, Schmittgen TD (2001) Analysis of relative gene expression data using real-time quantitative PCR and the 2(-Delta Delta C(T)) Method. Methods 25: $402-408$

Modric T, Silha JV, Shi Z, Gui Y, Suwanichkul A, Durham SK, Powell DR, Murphy LJ (2001) Phenotypic manifestations of insulin-like growth factor-binding protein-3 overexpression in transgenic mice. Endocrinology 142: $1958-1967$

Mummaneni P, Walker KA, Bishop PL, Turker MS (1995) Epigenetic gene inactivation induced by a cis-acting methylation center. J Biol Chem 270: $788-792$

Nakayama M, Bennett CJ, Hicks JL, Epstein JI, Platz EA, Nelson WG, De Marzo AM (2003) Hypermethylation of the human glutathione $S$-transferase-pi gene (GSTP1) CpG island is present in a subset of proliferative inflammatory atrophy lesions but not in normal or hyperplastic epithelium of the prostate: a detailed study using lasercapture microdissection. Am J Pathol 163: 923-933

Nickerson T, Chang F, Lorimer D, Smeekens SP, Sawyers CL, Pollak M (2001) In vivo progression of LAPC-9 and LNCaP prostate cancer models to androgen independence is associated with increased expression of insulin-like growth factor I (IGF-I) and IGF-I receptor (IGF-IR). Cancer Res 61: 6276-6280

Nosaka K, Maeda M, Tamiya S, Sakai T, Mitsuya H, Matsuoka M (2000) Increasing methylation of the CDKN2A gene is associated with the progression of adult T-cell leukemia. Cancer Res 60: 1043-1048

Oh Y, Muller HL, Lamson G, Rosenfeld RG (1993) Insulin-like growth factor (IGF)-independent action of IGF-binding protein-3 in Hs578T human breast cancer cells. Cell surface binding and growth inhibition. J Biol Chem 268: $14964-14971$

Papatsoris AG, Karamouzis MV, Papavassiliou AG (2005) Novel insights into the implication of the IGF-1 network in prostate cancer. Trends Mol Med 11: $52-55$

Perry AS, Foley R, Woodson K, Lawler M (2006) The emerging roles of DNA methylation in the clinical management of prostate cancer. Endocr Relat Cancer 13: $357-377$

Prestridge DS (1995) Predicting Pol II promoter sequences using transcription factor binding sites. J Mol Biol 249: $923-932$

Rebhan M, Chalifa-Caspi V, Prilusky J, Lancet D (1997) GeneCards: integrating information about genes, proteins and diseases. Trends Genet 13: 163
Renehan AG, Zwahlen M, Minder C, O’Dwyer ST, Shalet SM, Egger M (2004) Insulin-like growth factor (IGF)-I, IGF binding protein-3, and cancer risk: systematic review and meta-regression analysis. Lancet 363: $1346-1353$

Su AI, Welsh JB, Sapinoso LM, Kern SG, Dimitrov P, Lapp H, Schultz PG, Powell SM, Moskaluk CA, Frierson Jr HF, Hampton GM (2001) Molecular classification of human carcinomas by use of gene expression signatures. Cancer Res 61: 7388-7393

Takai D, Jones PA (2002) Comprehensive analysis of CpG islands in human chromosomes 21 and 22. Proc Natl Acad Sci USA 99: $3740-3745$

Thelen P, Burfeind P, Grzmil M, Voigt S, Ringert RH, Hemmerlein B (2004) cDNA microarray analysis with amplified RNA after isolation of intact cellular RNA from neoplastic and non-neoplastic prostate tissue separated by laser microdissections. Int J Oncol 24: 1085-1092

Thrasher JB, Tennant MK, Twomey PA, Hansberry KL, Wettlaufer JN, Plymate SR (1996) Immunohistochemical localization of insulin-like growth factor binding proteins 2 and 3 in prostate tissue: clinical correlations. J Urol 155: $999-1003$

Wang Y, Leung FC (2004) An evaluation of new criteria for CpG islands in the human genome as gene markers. Bioinformatics 20: $1170-1177$

Wheeler DL, Church DM, Lash AE, Leipe DD, Madden TL, Pontius JU, Schuler GD, Schriml LM, Tatusova TA, Wagner L, Rapp BA (2001) Database resources of the National Center for Biotechnology Information. Nucleic Acids Res 29: $11-16$

Wiley A, Katsaros D, Chen H, Rigault de la Longrais IA, Beeghly A, Puopolo M, Singal R, Zhang Y, Amoako A, Zelterman D, Yu H (2006) Aberrant promoter methylation of multiple genes in malignant ovarian tumors and in ovarian tumors with low malignant potential. Cancer 107: $299-308$

Yamanaka M, Watanabe M, Yamada Y, Takagi A, Murata T, Takahashi H, Suzuki H, Ito H, Tsukino H, Katoh T, Sugimura Y, Shiraishi T (2003) Altered methylation of multiple genes in carcinogenesis of the prostate. Int J Cancer 106: $382-387$

Yegnasubramanian S, Kowalski J, Gonzalgo ML, Zahurak M, Piantadosi S, Walsh PC, Bova GS, De Marzo AM, Isaacs WB, Nelson WG (2004) Hypermethylation of $\mathrm{CpG}$ islands in primary and metastatic human prostate cancer. Cancer Res 64: 1975-1986 